\title{
Septic versus aseptic hip revision: how different?
}

\author{
Carlo Luca Romanò • Delia Romanò • \\ Nicola Logoluso $\cdot$ Enzo Meani
}

Received: 1 February 2010/Accepted: 12 August 2010/Published online: 2 September 2010

(C) The Author(s) 2010. This article is published with open access at Springerlink.com

\begin{abstract}
Background The few available studies directly comparing aseptic and septic joint revision surgery report conflicting results. We investigated whether two-stage revision of septic hip prosthesis with a preformed antibiotic-loaded spacer and an uncemented prosthesis provides hip function and quality of life similar to those provided by aseptic revision surgery in the medium term, as well as the associated direct hospital costs.

Materials and methods We prospectively evaluated the hip function (Harris hip score) and quality of life (WOMAC and SF-12 scores) of 80 patients who underwent one-stage revision for aseptic loosening (Group A, 40 patients) or two-stage revision for septic total hip prostheses (Group S, 40 patients). Patients were matched for gender, age, and bone loss. A preformed antibiotic-loaded cement spacer was used for two-stage revision, and uncemented modular prostheses were implanted at revision in both groups. The minimum follow-up was 2 years (average 4 years; range 2-6 years).

Results We found no difference in infection recurrence or aseptic loosening rate in the two groups. Average Harris hip score increased similarly in both groups: from 19.1 to 74.0 in Group A versus 15.0-71.2 in Group S. Patient-reported quality-of-life questionnaires (SF-12 and WOMAC) at last
\end{abstract}

C. L. Romanò $(\bowtie) \cdot$ D. Romanò · N. Logoluso

Dipartimento di Chirurgia Ricostruttiva e delle Infezioni

Osteo-articolari, Istituto Ortopedico IRCCS Galeazzi,

Via Riccardo Galeazzi, 4-20166 Milan, Italy

e-mail: carlo.romano@grupposandonato.it

E. Meani

Centro di Chirurgia Osteo-articolare Settica,

Istituto Ortopedico Gaetano Pini, Milan, Italy follow-up were similar postoperatively, but the complication rate for Group S was twice that of Group A (20.8 versus $10 \%)$. Mean overall hospital-related costs of two-stage procedures were 2.2 times greater than those for aseptic revisions.

Conclusions Two-stage revision for infected hip prostheses, using a preformed antibiotic-loaded cement spacer and uncemented revision prosthesis, offers a success rate comparable to noninfected revisions in the medium term but is associated with a higher complication rate and costs.

Keywords Hip · Infection · Revision · Two-stage . Outcome

\section{Introduction}

Joint prosthesis infection has been recently reported as the third most common reason for revision in the United States [5] after instability/dislocation and aseptic mechanical loosening. Two-stage revision is the most widely accepted and performed intervention for septic hip prostheses, with an infection eradication rate exceeding $90 \%$ in most studies $[3,9,10,13,17,20,23,27,28,31,35,36]$. Despite its ability to control infection, the functional outcomes of revision surgery of septic cases are reportedly lower than those from aseptic revision, as described by Barrack et al. [4] and Wang et al. [33]. However, no previous study has directly compared two-stage revision of septic hip prosthesis with aseptic revision.

We therefore investigated whether patients undergoing two-stage revision for septic hip prosthesis or aseptic hip revision had similar (1) similar functional scores, (2) selfreported qualities of life, (3) postoperative infection and complication rates, and (4) radiographic signs of prosthetic 
loosening and rerevision rates. We also compared (5) the direct hospital costs connected with the two procedures.

\section{Materials and methods}

We prospectively followed 40 patients who underwent onestage revision for aseptic loosening (Group A) and 40 who underwent two-stage revision for septic total hip prosthesis (Group S) from 2001 to 2006. Sample size determination was carried out on the basis of the mean values and standard deviations that have been reported for the functional scores (WOMAC score) associated with septic and aseptic revision of total knee arthroplasty [24]. A sample size of approximately 35 patients per group was needed to achieve $90 \%$ power to detect differences of $10 \%$ between groups assuming a pooled standard deviation of $20 \%$ using the unpaired Student's $t$ test. Patients were matched for gender, age, and bone loss (Table 1). There was no difference $(P=0.31)$ between the mean \pm SD preoperative Harris hip scores of Groups A (19.1 \pm 18.5 ; range 12-62) and $S$ (15.0 \pm 17.7 ; range 12-50). Six patients in Group A and 9 in Group $S$ had had one previous revision surgery. Three patients were lost to follow-up: two in Group A and one in Group S; therefore, a total of 77 hips were available for follow-up at a minimum of 2 years (mean, 4 years; range 2-6 years). The study was approved by the local Institutional Review Board and Ethics Committee. All of the patients provided informed consent for enrollment in the study, which conformed to the Declaration of Helsinki.

We classified patients according to the Cierny-Mader classification [7]. In this classification, hosts affected by bone infection are divided into three classes: Type A hosts have a normal, uncompromised, immune system; Type B are locally and/or systemically immunocompromised hosts; and Type $\mathrm{C}$ are patients that are not considered surgical candidates (surgical treatment is more compromising to the patient than the disability caused by the disease itself). There were more Type B hosts in Group $S$ than in Group A: 20 of 40 in Group $S$ and 8 of 40 patients in Group A.

Aseptic loosening was confirmed in all cases by preoperative C-reactive protein (CRP) and erythrocyte sedimentation rate (ESR) values below or equal to, respectively, $10 \mathrm{mg} / \mathrm{l}$ and $20 \mathrm{~mm} / \mathrm{h}$, and negative intraoperative cultures [15]. The reasons for aseptic revision were mechanical loosening of components resulting from polyethylene wear and osteolysis (35 hips), metallosis (two cases), and recurrent dislocation (3 hips). In Group A, the average time from the initial THA to one-stage revision was $71 \pm 28$ months (range 15-161 months). The initial diagnosis had been primary osteoarthritis in 22 patients, hip dysplasia in 12, femoral head necrosis in 4 , and femoral neck fracture in 2 patients.

Diagnosis of infection in the patients included in Group $\mathrm{S}$ was based on the presence of one or more of the following conditions: (1) a draining fistula; (2) positive intraoperative cultures in at least two samples out of five; (3) positive histologic findings and preoperative elevated CRP values (greater than $15 \mathrm{mg} / \mathrm{l}$ ). Samples for frozensection analysis and permanent histological analysis were obtained from the pseudocapsule, the membrane around the prosthesis, or tissue that was suspected of being infected. Each of two samples from each patient were divided into two parts, one for frozen-section analysis and one for

Table 1 Patients details

\begin{tabular}{llll}
\hline Preoperative data & Group A & Group S & $P$ value \\
\hline Number of patients & 40 & 40 & \\
Male/female & $26 / 14$ & $22 / 18$ & 0.5 \\
Average age (range) & $64.4 \pm 6.4$ & $65.3 \pm 8.6$ & 0.06 \\
Body weight (kg) & $68.3 \pm 7.5$ & $64.9 \pm 7.3$ & 0.08 \\
Body height (cm) & $163.5 \pm 9.4$ & $160.1 \pm 8.8$ & 0.01 \\
Type B hosts & 8 & 20 & 0.3 \\
Harris hip score & $19.1 \pm 18.5$ (range 12-62) & $15.0 \pm 17.7$ (range 12-50) & 0.4 \\
Previous revisions & $0.15 \pm 0.36$ & $0.22 \pm 0.42$ & 0.5 \\
Follow-up (months) & $50.5 \pm 11.8$ & $52.1 \pm 11.7$ & \\
Bone loss (Paprosky) & & & \\
Acetabulum type 1 & 5 & 4 & \\
Acetabulum type 2 & 22 & 20 & \\
Acetabulum type 3 & 13 & 16 & \\
Femur type 1 & 2 & 1 & \\
Femur type 2 & 8 & 6 & \\
Femur type 3 & 18 & 22 & \\
Femur type 4 & 12 & 11 & \\
\hline
\end{tabular}


permanent paraffin-embedded section analysis. The samples that were used for frozen-section analysis were snapfrozen in carbon dioxide; $4 \mu \mathrm{m}$ sections were then cut and stained with hematoxylin and eosin. The samples used for histological analysis of paraffin-embedded sections were fixed in formalin and embedded in paraffin prior to staining with hematoxylin and eosin. The most cellular areas in the tissue sample were chosen for evaluation, and the number of neutrophils (in the frozen and paraffin-embedded sections), lymphocytes, and plasma cells (in the paraffinembedded sections) per high-power field $(400 \times)$ in at least ten separate microscopic fields were counted. The histological Feldman criterion, defined as the presence of at least five neutrophils per high-power field $(400 \times)$ in at least five separate microscopic fields, was used [2, 14, 19].

The average time from the initial THA to the revision in Group S was $29 \pm 28$ months (range 8-61 months). Seventy percent of the isolated Staphylococci, the most frequently cultured pathogen in patients from Group $\mathrm{S}$, were oxacillin resistant (Table 2). Bacteriologic examinations of four hips found no growth, in which case the diagnosis of infection was made by clinical findings and histologic examination.

All patients were operated on through a lateral approach with the patient lying in the supine position, and all underwent revision of both hip prosthetic components.

In Group S, the infected hip prosthesis was removed and a preformed antibiotic-loaded spacer (InterSpace ${ }^{\circledR}$ Hip; Tecres SpA, Verona, Italy; Exactech Inc., Gainesville, FL, USA) was implanted. The InterSpace ${ }^{\circledR}$ Hip is an off-theshelf polymethylmethacrylate antibiotic-loaded preformed hip spacer. The inner part of the spacer features a stainless still rod that increases mechanical resistance. The cement is preloaded by the manufacturer with gentamicin at a concentration of $1.9 \%$. The InterSpace ${ }^{\circledR}$ Hip is available with three different head sizes and with two stem sizes, short $(260 \mathrm{~mm})$ and long $(360 \mathrm{~mm})$, that may be chosen intraoperatively (Fig. 1). The spacer was fixed only in the

Table 2 Organisms cultured in the septic group of 40 patients

\begin{tabular}{ll}
\hline Isolated microorganism & Number \\
\hline Staphylococcus epidermidis & 16 \\
Staphylococcus aureus & 15 \\
Oxacillin-resistant Staphylococci & 22 \\
Streptococcus & 4 \\
Pseudomonas aeruginosa & 4 \\
Enterococcus & 4 \\
Peptostreptococcus & 2 \\
Propionibacterium & 2 \\
Serratia liquefaciens & 1 \\
Corynebacterium & 1 \\
\hline
\end{tabular}

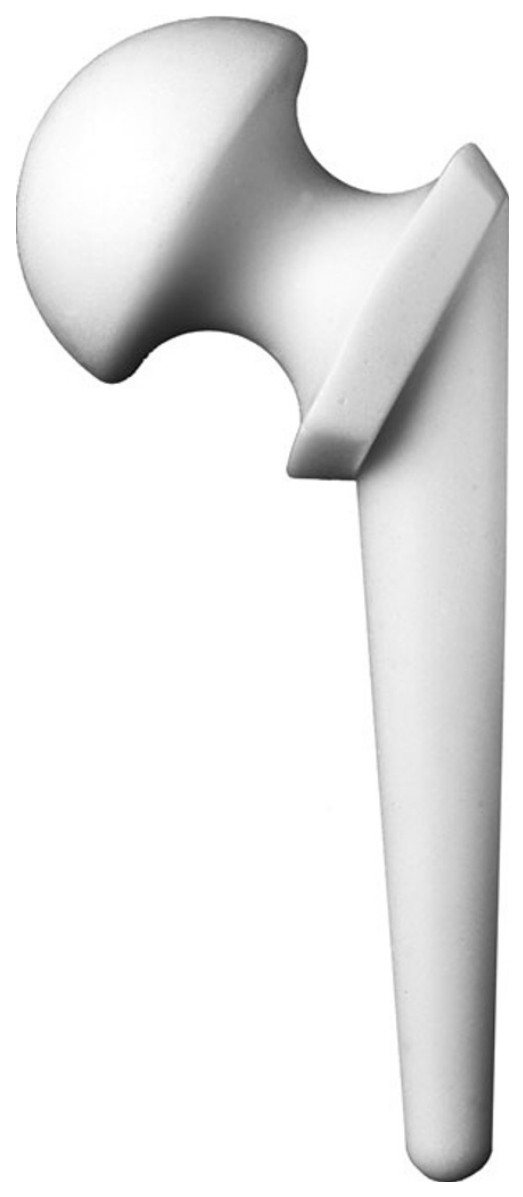

Fig. 1 The preformed antibiotic-loaded spacer used in the study for two-stage hip revision (InterSpace ${ }^{\circledR}$ Hip; Tecres SpA, Verona, Italy; Exactech Inc., Gainesville, FL, USA). The spacer comes in three different head sizes and two stem sizes: short $(260 \mathrm{~mm}$, shown in figure) and long $(360 \mathrm{~mm})$. The size may be chosen intraoperatively by the surgeon on the basis of reusable trials

proximal part (Fig. 2), to prevent implant rotation, with one pack of antibiotic-loaded cement (Cemex Genta; Tecres Spa) containing gentamicin $1.9 \%$ and vancomycin $5 \%$. The vancomycin powder was thoroughly mixed with the cement powder into a fine consistency before the addition of liquid monomer. Cement mixing was performed without vacuum. No bone grafts were used at the time of spacer implantation.

After surgery, systemic antibiotics were administered for 4-6 weeks on the basis of antibiogram, when available. The most commonly used antibiotic regime was a combination of two parenteral antibiotics (a glycopeptide and a carbapenemic) for 2 weeks followed by a combination of two oral antibiotics (rifampicin and a fluoroquinolone) for the remaining 2-4 weeks. Patients were allowed to sit in a chair on the second day after operation and partial weightbearing (10-15 kg on the operated leg) with two crutches from the third postoperative day until revision surgery. Isometric and isotonic exercises were usually 


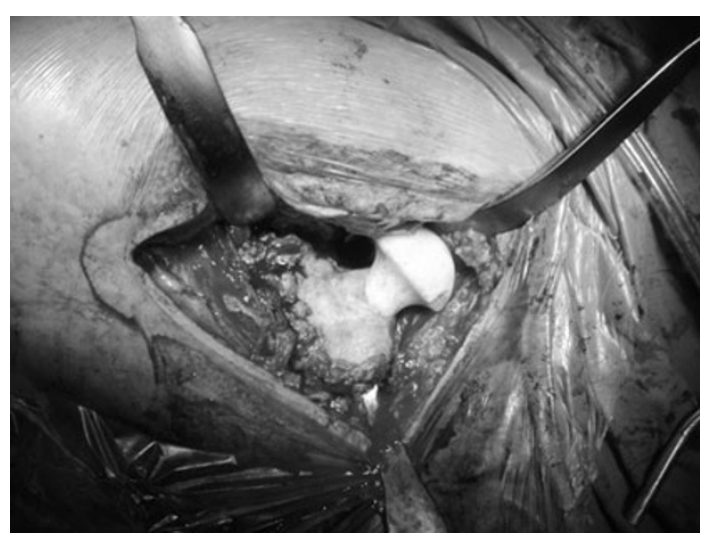

Fig. 2 An intraoperative photograph shows that the preformed cement spacer is only proximally fixed with antibiotic-loaded cement to prevent rotational instability and unwanted sinking of the implant

continued in a rehabilitation center within our hospital until the 20th postoperative day.

Follow-up included blood testing (cell count with differential, CRP, ESR, liver and renal function) every 2 weeks and clinical examination with a plain radiograph of the hip and femur 45 days after surgery. Patients with successful eradication of their infection, as evidenced clinically and by a complete blood count with differential and CRP within the normal ranges, underwent the second stage of their reconstruction. If clinical suspicion of persistent infection remained, joint aspiration before reimplantation was performed for cultures and white blood cell count. In all cases, intraoperative cultures were obtained at the time of the second-stage procedure. At revision, the hip was exposed through the same lateral incision and the spacers were removed. Reimplantation was performed 9-16 weeks after the spacer implantation.

In both groups (A and S), revision surgery was performed with modular titanium cementless femoral components (PROFEMUR ${ }^{\circledR}$ Hip System; Wright Medical Group Inc., Arlington, TN, USA, or S-ROM; DePuy Orthopaedics Inc., Warsaw, IN, USA). Unconstrained cementless acetabular components were used in all cases. Eight hips in Group A and 6 in Group S had cancellous bone grafts for bone defects.

Touchdown weightbearing was allowed for 6 weeks, followed by $50 \%$ weightbearing for 6 weeks. Full weightbearing and abductor strengthening were permitted 12 weeks after surgery. After each procedure, closed suction drainage was inserted and removed after $48 \mathrm{~h}$. All patients received $0.4 \mathrm{~mL}$ enoxaparin per day for 30 days after surgery to prevent thromboembolic complications and $200 \mathrm{mg}$ celecoxib twice a day for 10-14 days after revision surgery to prevent heterotopic ossifications [30].

Clinical and radiographic assessments were performed by an independent observer (DR) who was not associated with the treatment preoperatively, at the time of spacer removal, and at the latest follow-up after reimplantation. Bone defect classification, according to Paprosky [8], was performed intraoperatively by the surgeon at the time of spacer implantation and at revision. Hip function was recorded using the Harris hip score [13]. Clinical signs of infection (redness, swelling, pain, fistulae) and other complications (deep venous thrombosis and/or pulmonary embolism, postoperative hemorrhage, dislocation, and nerve palsy) were also recorded at follow-up. Laboratory tests at each visit consisted of a complete blood count with differential, ESR, CRP, urea and creatinine, and a creatinine clearance test.

Patient-reported quality-of-life (QOL) outcomes were assessed pre- and postoperatively through an Italian translation of the WOMAC questionnaire evaluated at its subscales [18]. Each raw score was transformed into a 0-100 scale, with 0 being the worst QOL and 100 the best QOL. An Italian translation of the SF-12 questionnaire $[1,34]$ was also administered to all the patients, but only postoperatively.

Plain radiographs included anteroposterior and lateral views of the hip. Radiographic examination was performed preoperatively, at spacer removal, at reimplantation, and then at 3,6 , and 12 months postoperatively, followed by yearly intervals thereafter.

Primary outcomes included (1) Harris hip score, (2) patient-reported QOL evaluated through the SF-12 and WOMAC questionnaires, (3) postoperative infection and complication rates, and (4) radiographic signs of prosthetic loosening and rerevision rate. Secondary outcome was an estimate of the direct hospital costs of the two procedures. To this end, we performed a retrospective cost identification study on the two cohorts of patients, Groups A and S. Data on resource use included costs of operating room equipment and implants (including bone grafts or bone substitutes), operative staff and time, hospital stay in the surgical and rehabilitation departments, blood, pharmacy and administrative costs per patient, as extracted from the hospital administrative decision support database.

The differences in the Harris hip score, WOMAC and SF-12 scores, leg length discrepancy, and hospital costs between Groups $\mathrm{A}$ and $\mathrm{S}$ were determined using the unpaired Student's $t$ test. The complication rate difference between groups, considered a categorical variable, was analyzed by the two-tailed Fisher's exact test. The software used for statistical analysis was GraphPad InStat from GraphPad Software, Inc. (San Diego, CA, USA).

\section{Results}

At a mean follow-up of 4 years postoperatively, the Harris hip scores increased to, respectively, $74.0 \pm 13.4$ (range 
Table 3 WOMAC scores

\begin{tabular}{llll}
\hline Preoperative data & Group A $(N=40)$ & Group S $(N=40)$ & $P$ value \\
\hline Function & $41.7 \pm 22.3$ & $56.7 \pm 17.8$ & 0.001 \\
Pain & $43.9 \pm 17.9$ & $52.6 \pm 22.7$ & 0.06 \\
Stiffness & $41.2 \pm 22.9$ & $44.9 \pm 23.8$ & 0.48 \\
\hline Postoperative data & Group A $(N=38)$ & Group S $(N=39)$ & $P$ \\
\hline Function & $66.2 \pm 22.3$ & $76.6 \pm 21.3$ & 0.04 \\
Pain & $75.8 \pm 24.0$ & $77.4 \pm 22.8$ & 0.76 \\
Stiffness & $70.1 \pm 22.5$ & $71.4 \pm 24.1$ & 0.80 \\
\hline
\end{tabular}

61-88) and $71.2 \pm 14.1$ (range 55-86) $(P=0.37)$ in Groups $\mathrm{A}$ and $\mathrm{S}$. Average leg length discrepancy was $1.3 \pm 0.9 \mathrm{~cm}$ in Group $\mathrm{A}$ and $1.5 \pm 1.0 \mathrm{~cm}$ in Group $\mathrm{S}$ $(P=0.35)$.

The physical functional domain of the WOMAC score was better for Group $S$ than for Group A both pre- and postoperatively. On average, Group A patients reported more pain preoperatively than patients in Group $S$; this difference was no longer present after revision (Table 3).

The mean SF-12 score at last follow-up was similar for the two groups for the physical $(P=0.09)$ and the mental well-being $(P=0.25)$ components (Group S: mental component score: $43.1 \pm 13.8$, physical component score: $35.6 \pm 12.4$; Group A: $48.7 \pm 14.9$ and $32.2 \pm 13.4$, respectively).

The postoperative infection rates were similar, with one deep infection in each group that required further surgery within 2 years of revision. There were 5 intraoperative complications. Two patients in Group A had a small split in the proximal femoral shaft, which was treated with a cerclage wire. Three patients in Group $S$ had a crack in the greater trochanter (two at prosthesis removal and spacer insertion and another one at reimplantation), which required no treatment. Seven patients had complications during the perioperative period.

One patient in Group $\mathrm{S}$ had postoperative bleeding that required the draining of a hematoma, the stapling of minor vessels and the use of hemostatic agents. One in Group A and two in Group $S$ had a deep vein thrombosis diagnosed on routine postoperative Doppler ultrasound; in one patient in Group S, it was above the knee and resulted in a pulmonary embolus. One patient in Group S had transient peroneal nerve palsy and one patient in each group had hip dislocation, which was treated surgically in the patient in Group S by changing the modular neck of the prosthesis. The overall complication rate, including intraoperative complications, postoperative deep venous thrombosis and/or pulmonary embolism, postoperative hemorrhage, dislocation and nerve palsy, was greater in Group $\mathrm{S}$ than in Group A: 4 of 40 (10\%) patients in Group A had complications, and 9 of 40 (20.8\%) in Group S. No femoral components or acetabular cups were radiographically loosened at the latest follow-up. However, three patients in Group A and two in Group S had osteolysis in femoral Gruen Zone 1, and two more had osteolysis in Zone 7. One more femoral component in Group $S$ required revision at 2 years for aseptic subsidence.

Mean overall hospital-related costs of two-stage revision of septic THAs $(60,394 \pm 15,886$ euros $)$ were 2.2 times greater than those associated with aseptic revision $(27,194 \pm 5,122$ euros) (Table 4). Only pharmaceutical costs were similar between the two groups.

\section{Discussion}

Although patient satisfaction and quality of life improvements have been shown by different authors to be better after primary than after revision surgery in the hip [11, 12, 25, 29], little is known from prospective comparative studies about patient function and quality of life and the reasons for THA revision. On the other hand, conflicting results have been reported upon comparing septic and aseptic TKA revision, with some papers showing inferior [4, 33] and others superior [24, 26] knee scores and/or patient satisfaction and quality of life for two-stage septic versus aseptic TKA revision surgery. In this study, we compared the medium-term hip functions, QOLs, and complication rates of two cohorts of patients operated on for aseptic or septic THA revision.

Our study has several limitations. First, the preoperative comorbidities differed among the two groups, with more B hosts in the septic patients. This is not an unexpected finding due to the relative odds ratio for different comorbidities with respect to the risk of postoperative infection, and it represents a bias that is difficult to completely overcome when comparing populations of patients with and without septic complications. Considering the limited number of patients, it was not possible to assess if there were different postoperative morbidities or direct medical costs for the A and B hosts within each group. Second, the follow-up is relatively short, and more prolonged observation is needed to provide information on long-term implant survivorship and occurrence of infection in the two groups. Third, our analysis of direct medical costs did not consider readmissions, outpatient visits and charges, or nonmedical or indirect costs to the patient and to society associated with lost productivity. Considering the prolonged period of convalescence usually associated with septic THA revision, the inclusion of these other costs would probably strengthen the conclusion that septic revision is associated with greater resource use than revision for aseptic loosening. Caution should also be applied 
Table 4 Mean direct hospital costs (in euros) per patient \pm SD

\begin{tabular}{lrrrl}
\hline Hospital resource & \multicolumn{1}{l}{ Group A } & \multicolumn{1}{c}{ Group S } & Group S/Group A & $P$ value \\
\hline Operating room equipment and implants & $10,620 \pm 5938$ & $18,367 \pm 9029$ & 1.7 & 0.0001 \\
Operative time and staff & $4529 \pm 1522$ & $9458 \pm 7533$ & 2.1 & 0.0001 \\
Hospital stay & $6901 \pm 4796$ & $21,442 \pm 7522$ & 3.1 & 0.0001 \\
Material services & $2122 \pm 1285$ & $5284 \pm 1782$ & 2.5 & 0.0001 \\
Blood products & $1668 \pm 1980$ & $2421 \pm 1208$ & 1.4 & 0.04 \\
Pharmacy & $1354 \pm 6076$ & $3422 \pm 6584$ & 2.5 & 0.14 \\
Total & $27,194 \pm 5122$ & $60,394 \pm 15886$ & 2.2 & 0.0001 \\
\hline
\end{tabular}

when generalizing those data, since treatment regimes and resource valorization may vary among countries and hospitals.

Two-stage reimplantation with a cement-impregnated spacer remains the gold standard and the most commonly performed intervention for patients with infected hip arthroplasty. Our data show that hip function, as determined through the Harris hip score, was similar in the two cohorts of patients studied, notwithstanding the reason for revision. The WOMAC score is a reliable and sensitive tool for measuring QOL and outcome after THA [21, 22]. We found better functional WOMAC scores both pre- and postoperatively in Group S compared with Group A. One possible explanation for this is the occurrence of more patients with septic complications that came to our attention with otherwise well-fixed and relatively pain-free prostheses. In line with this interpretation is the finding of an average lower self-reported preoperative pain score in patients in Group S than in Group A. In a paper comparing aseptic cases of knee prosthetic revision with two-stage revision of septic knees using an articulated spacer, Meek et al. [24] reported that two-stage exchange provided comparable patient satisfaction and functional results (WOMAC, Oxford-12, SF-12, patient satisfaction data and range of motion). The authors noted "statistically better postoperative function scores for the septic group," similar to those observed in our hip study, and suggested that "perhaps it is not that the septic revisions are doing better than expected, but that the aseptic revisions may often do worse than is presently assumed." Similar findings have been more recently reported by Patil and coworkers [26].

Our study also shows similar infection recurrence rates in the two groups at medium-term follow-up, whereas the overall complication rate after septic surgery is approximately twice that after aseptic revision. We interpret this latter finding as the consequence of submitting the patients to two procedures instead of one without any substantial additional risk of complication resulting from infection itself. In this regard, our data are slightly different from those reported by Bozic and coworkers [6], who showed a 2.7-fold increase in the complication rate in patients operated on for two-stage hip revision compared with one-stage aseptic cases, suggesting an additional infection risk from two-stage procedures compared with one-stage for aseptic cases.

Direct medical costs associated with revision THA because of aseptic loosening were reportedly up to 4.8 -fold higher than the direct medical costs associated with primary THA [6]. Sculco [32] evaluated the economic impact of infection after total joint arthroplasty. Noting the disproportionate burden of care associated with septic hip revision surgery, he recommended that a method for case sharing should be established by medical centers that are best equipped to care for these patients, and that reimbursement to both hospitals and physicians should more realistically adapted to the magnitude of resources consumed by these patients. Direct medical costs associated with revision THA due to infection were 2.2 times higher than those for aseptic loosening in our study. This finding is in accordance with those described previously by other authors. Hebert et al. [16] reported that the surgical treatment of patients with an infection after TKA was approximately twice as expensive as that required for revision TKA because of aseptic loosening, whereas Bozic [6] reported that total direct medical costs associated with revision THA due to infection were 2.8 times higher than the direct medical costs associated with revision THA due to aseptic loosening. In this regard it should be noted that calculating the costs of two-stage versus one-stage aseptic revision is not just a matter of "two procedures versus one." While septic cases may require more extensive and lengthy surgery and costlier medical treatment, the firststage procedure is less expensive than a reimplantation procedure (the cost of the spacer, even if it is a preformed, off-the-shelf device, is much less than the cost of a revision prosthesis; the surgical time needed to implant a spacer is often less that that required to implant the revision femoral and acetabular prosthesis, etc.).

In conclusion, our data suggest that at medium-term follow-up, two-stage revision of septic hip arthroplasty with the use of a preformed antibiotic-loaded cement spacer and a cementless modular revision prosthesis may provide functional results, as the Harris hip score and QOL improvements were comparable to those obtained after revision for aseptic loosening. The higher complication rate 
and the additional resource use required to provide care for patients with an infection after total joint arthroplasty should prompt healthcare systems to provide adequate reimbursements and/or to develop specialized centers with dedicated, appropriate funding to better manage these challenging cases.

Acknowledgments Each author certifies that his or her institution approved the human protocol for this investigation and that all investigations were conducted in conformity with ethical principles of research. This work was performed at IRCCS Istituto Ortopedico Galeazzi, Centro di Chirurgia Ricostruttiva e delle Infezioni Osteoarticolari, Milan, Italy, and Istituto Ortopedico Gaetano Pini, Centro Complicanze Ortopediche Settiche, Milan, Italy.

\section{Conflict of interest None.}

Open Access This article is distributed under the terms of the Creative Commons Attribution Noncommercial License which permits any noncommercial use, distribution, and reproduction in any medium, provided the original author(s) and source are credited.

\section{References}

1. Apolone G, Mosconi P Quattrociocchi L, Granicolo EA, Groth NJE (2009) SF-12 health status questionnaire (Italian version). http://crc.marionegri.it/qdv/downloads/SF12\%20Manuale.pdf. Accessed 12 Dec 2009

2. Athanasou NA, Pandey R, de Steiger R, McLardy Smith P (1997) The role of intraoperative frozen sections in revision total joint arthroplasty. J Bone Joint Surg Am 79:1433-1434

3. Barrack RL (2002) Rush pin technique for temporary antibioticimpregnated cement prosthesis for infected total hip arthroplasty. J Arthroplasty 17:600-603

4. Barrack RL, Engh G, Rorabeck C, Sawhney J, Woolfrey M (2000) Patient satisfaction and outcome after septic versus aseptic revision total knee arthroplasty. J Arthroplasty 15:990-993

5. Bozic KJ, Kurtz SM, Lau E, Ong K, Vail TP, Berry DJ (2009) The epidemiology of revision total hip arthroplasty in the United States. J Bone Joint Surg Am 91:128-133

6. Bozic KJ, Ries MD (2005) The impact of infection after total hip arthroplasty on hospital and surgeon resource utilization. J Bone Joint Surg Am 87:1746-1751

7. Cierny G III, Pennick JJ, Mader JT (2003) A clinical staging system for adult osteomyelitis. Clin Orthop Relat Res 414:7-24

8. Della Valle CJ, Paprosky WG (2003) Classification and an algorithmic approach to the reconstruction of femoral deficiency in revision total hip arthroplasty. J Bone Joint Surg Am 85(Suppl 4):1-6

9. Duncan CP, Masri BA (1995) The role of antibiotic-loaded cement in the treatment of an infection after a hip replacement. Instr Course Lect 44:305-313

10. Durbhakula SM, Czajka J, Fuchs MD, Uhl RL (2004) Spacer endoprosthesis for the treatment of infected total hip arthroplasty. J Arthroplasty 19:760-767

11. Eisler T, Svensson O, Tengström A, Elmstedt E (2002) Patient expectation and satisfaction in revision total hip arthroplasty. J Arthroplasty 17:457-462

12. Espehaug B, Havelin LI, Engesaeter LB, Langeland N, Vollset SE (1998) Patient satisfaction and function after primary and revision total hip replacement. Clin Orthop Relat Res 351:135-148
13. Etienne G, Waldman B, Rajadhyaksha AD, Ragland PS, Mont MA (2003) Use of a functional temporary prosthesis in a twostage approach to infection at the site of a total hip arthroplasty. J Bone Joint Surg Am 85(Suppl 4):94-96

14. Feldman DS, Lonner JH, Desai P, Zuckerman JD (1995) The role of intraoperative frozen sections in revision total joint arthroplasty. J Bone Joint Surg Am 77:1807-1813

15. Greidanus NV, Masri BA, Garbuz DS, Wilson SD, McAlinden MG, Xu M, Duncan CP (2007) Use of erythrocyte sedimentation rate and C-reactive protein level to diagnose infection before revision total knee arthroplasty a prospective evaluation. J Bone Joint Surg Am 89:1409-1416

16. Hebert CK, Williams RE, Levy RS, Barrack RL (1996) Cost of treating an infected total knee replacement. Clin Orthop Relat Res 331:140-145

17. Hsieh $\mathrm{PH}$, Chen LH, Chen $\mathrm{CH}$, Lee MS, Yang WE, Shih $\mathrm{CH}$ (2004) Two-stage revision hip arthroplasty for infection with a custom-made, antibiotic-loaded, cement prosthesis as an interim spacer. J Trauma 56:1247-1252

18. Klassbo M, Larsson E, Mannevik E (2003) Hip disability and osteoarthritis outcome score. An extension of the Western Ontario and McMaster Universities Osteoarthritis Index. Scand J Rheumatol 32:46-51

19. Lonner JH, Desai P, Dicesare PE, Steiner G, Zuckerman JD (1996) The reliability of analysis of intraoperative frozen sections for identifying active infection during revision hip or knee arthroplasty. J Bone Joint Surg 78A:1553-1558

20. Magnan B, Regis D, Biscaglia R, Bartolozzi P (2001) Preformed acrylic bone cement spacer loaded with antibiotics: use of twostage procedure in 10 patients because of infected hips after total replacement. Acta Orthop Scand 72:591-594

21. Martin DP, Engelberg R, Agel J, Swiontkowski MF (1997) Comparison of the musculoskeletal function assessment questionnaire with the Short Form-36, the Western Ontario and McMaster Universities Osteoarthritis index, and the sickness impact profile health-status measures. J Bone Joint Surg Am 79:1323-1325

22. McGrory BJ, Harris WH (1996) Can the Western Ontario and McMaster Universities (WOMAC) Osteoarthritis Index be used to evaluate different hip joints in the same patients? J Arthroplasty 11:841

23. Meani E, Romanò CL, Crosby L, Hofmann G (2007) Infection and local treatment in orthopaedic infections. Springer, Heidelberg

24. Meek RM, Dunlop D, Garbuz DS, McGraw R, Greidanus NV, Masri BA (2004) Patient satisfaction and functional status after aseptic versus septic revision total knee arthroplasty using the PROSTALAC articulating spacer. J Arthroplasty 19:874-879

25. Patil S, Garbuz DS, Greidanus NV, Masri BA, Duncan CP (2008) Quality of life outcomes in revision vs primary total hip arthroplasty: a prospective cohort study. J Arthroplasty 23:550-553

26. Patil S, Lee K, Huddleston JI, Harris AH, Goodman SB (2010) Aseptic versus septic revision total knee arthroplasty: patient satisfaction, outcome and quality of life improvement. Knee 17:200-203

27. Pearle AD, Sculco TP (2002) Technique for fabrication of an antibiotic-loaded cement hemiarthroplasty (ANTILOCH) prosthesis for infected total hip arthroplasty. Am J Orthop 31:425-427

28. Ries MD, Jergesen H (1999) An inexpensive molding method for antibiotic impregnated cement spacers in infected total hip arthroplasty. J Arthroplasty 14:764-765

29. Robinson AH, Palmer CR, Villar RN (1999) Is revision as good as primary hip replacement? A comparison of quality of life. J Bone Joint Surg Br 81:42 
30. Romano CL, Duci D, Romano D, Mazza M, Meani E (2004) Celecoxib versus indomethacin in the prevention of heterotopic ossification after total hip arthroplasty. J Arthroplasty 19:14

31. Schoellner C, Fuerderer S, Rompe JD, Eckardt A (2003) Individual bone cement spacers (IBCS) for septic hip revisionpreliminary report. Arch Orthop Trauma Surg 123:254-259

32. Sculco TP (1993) The economic impact of infected total joint arthroplasty. Instr Course Lect 42:349-351

33. Wang CJ, Hsieh MC, Huang TW, Wang JW, Chen HS, Liu CY (2004) Clinical outcome and patient satisfaction in aseptic and septic revision total knee arthroplasty. Knee 11:45-49
34. Ware JE, Kosinski M, Keller SD (1996) A 12-item short-form health survey: construction of scales and preliminary tests of reliability and validity. Med Care 34:0-233

35. Yamamoto K, Miyagawa N, Masaoka T, Katori Y, Shishido T, Imakiire A (2003) Clinical effectiveness of antibiotic-impregnated cement spacers for the treatment of infected implants of the hip joint. J Orthop Sci 8:823-828

36. Younger AS, Duncan CP, Masri BA, McGraw RW (1997) The outcome of two-stage arthroplasty using a custom-made interval spacer to treat the infected hip. J Arthroplasty 12:615-623 